\title{
Equal Sums of Quartics (In Context with the Richmond Equation) $\left(a x^{4}+b y^{4}+c z^{4}+d w^{4}=0\right)$
}

\author{
Seiji Tomita ${ }^{1}$, Oliver Couto ${ }^{2}$ \\ ${ }^{1}$ Tokyo Computer Company, Tokyo, Japan \\ ${ }^{2}$ University of Waterloo, Waterloo, Ontario, Canada
}

Received July 25, 2020; Revised October 12, 2020; Accepted October 24, 2020

\section{Cite This Paper in the following Citation Styles}

(a): [1] Seiji Tomita, Oliver Couto, "Equal Sums of Quartics (In Context with the Richmond Equation) $\left(a x^{4}+b y^{4}+c z^{4}+\right.$ $\left.d w^{4}=0\right)$," Universal Journal of Applied Mathematics, Vol. 8, No. 2, pp. 13 - 29, 2020. DOI: 10.13189/ujam.2020.080201.

(b): Seiji Tomita, Oliver Couto (2020). Equal Sums of Quartics (In Context with the Richmond Equation) $\left(a x^{4}+b y^{4}+c z^{4}+\right.$ $\left.d w^{4}=0\right)$. Universal Journal of Applied Mathematics, 8(2), 13 - 29. DOI: 10.13189/ujam.2020.080201.

Copyright $\bigcirc 2020$ by authors, all rights reserved. Authors agree that this article remains permanently open access under the terms of the Creative Commons Attribution License 4.0 International License

\begin{abstract}
Consider the below mentioned Equation: $a x^{4}+b y^{4}+c z^{4}+d w^{4}=0---[1]$ In section (1) we consider solution's with the condition on the coefficient's of equation[1]. Namely the product $(a b c d)=$ square. In section [2] we consider the coefficients of Equation [1], with the product of coefficient's (abcd) not equal to a square. Historically Equation [1] has been studied by Ajai Choudhry, A. Bremner, M.Ulas [ref. 5] in 2014. Also Richmond [ref. 1 $\& 2]$ has done some ground work in 1944 \& 1948 . This paper has gone a step further, by finding many parametric solutions \& new small numerical solutions by the use of unique Identities. The identities are unique, because they are of mixed powers (combination of quartic \& quadratic variables) which are then converted to only degree four identities. As an added bonus in section [B], we came up with a few quartic $(4-1-n)$ numerical solutions for $(n<50)$ by elliptical mean's. A table of numerical solutions for the (4-1-n) Equation arrived at by brute force computer search is also given [ref \# 7].
\end{abstract}

Keywords Quartic Equation, Diophantine Equation, Diagonal Quartic Surfaces, Number Theory

\section{Section (1)}

$$
a x^{4}+b y^{4}+c z^{4}+d w^{4}=0
$$

[Condition: The product $(a b c d)=$ square]

Existence of solution for diophantine equation

$$
a x^{4}+b y^{4}+c z^{4}+d w^{4}=0
$$

are known if abcd is square number.

Furthermore, condition of

$$
a+b+c+d=0
$$

or having one known solution is necessary to have a rational solution.

Anyway, we show some parametric solutions of

$a x^{4}+b y^{4}+c z^{4}+d w^{4}=0$ with abcd is square number. 


\section{Case (A)}

We show Diophantine Equation,

$$
2 m n X^{4}+\left(m^{2}-n^{2}\right) Y^{4}=2 m n Z^{4}+\left(m^{2}-n^{2}\right) W^{4}
$$

has infinitely many integer solutions. $\mathrm{m}, \mathrm{n}$ are arbitrary.

We consider below Equation:

Where, in Equation (1), ( a, b, c, d) $=(\mathrm{p}, \mathrm{q},-\mathrm{p},-\mathrm{q})$

$$
\begin{gathered}
p(a t+s)^{4}+q(b t-1)^{4}=p(a t-s)^{2}+q(b t+1)^{2} . \\
\left(8 p a^{3} s-8 q b^{3}\right) t^{3}+\left(8 p a s^{3}-8 q b\right) t=0
\end{gathered}
$$

To obtain a rational solution of Equation (2) for $t$, we have to find the rational solution of (3).

$$
v^{2}=-a^{4} s^{4} p^{2}+\left(q b^{3} a s^{3}+a^{3} s q b\right) p-q^{2} b^{4}
$$

Let consider Equation (3) is a quadratic Equation for $\mathrm{p}$.

$$
\begin{aligned}
& \text { Substitute } p=\frac{b}{s}, q=\text { a to equation (3), then } \\
& \qquad \begin{aligned}
& v^{2}=-a^{2} b^{2}(s-1)(s+1)(a-b)(a+b) . \\
& \text { Let } b= m^{2}+n^{2}, a=m^{2}-n^{2}, \\
& s=\frac{m^{2}+n^{2}}{2(m n)},
\end{aligned}
\end{aligned}
$$

then we obtain $p=2 m n$,

$$
\begin{aligned}
& q=m^{2}-n^{2}, \\
& v=\left(m^{2}+n^{2}\right)\left(m^{2}-n^{2}\right)^{2} \\
& t=\frac{n^{2}-m^{2}}{4\left(m^{2} n^{2}\right)} .
\end{aligned}
$$

Thus we obtain a parametric solution.

$$
\begin{gathered}
(p, q)=\left(2 m n, m^{2}-n^{2}\right) . \\
X=m^{4}-2 m^{2} n^{2}+n^{4}-2 m^{3} n-2 m n^{3} \\
Y=m^{4}-n^{4}+4 m^{2} n^{2} \\
Z=m^{4}-2 m^{2} n^{2}+n^{4}+2 m^{3} n+2 m n^{3} \\
W=m^{4}-n^{4}-4 m^{2} n^{2}
\end{gathered}
$$

Where, $\mathrm{m}, \mathrm{n}$ are arbitrary.

Furthermore, we can find other parametric solution by using this method.

Hence by repeating this process, we can obtain infinitely many integer solutions for Equation (1).

Example:

$$
\begin{gathered}
(m, n)=(2,1): \\
4 X^{4}+3 Y^{4}=4 Z^{4}+3 W^{4} \\
(X, Y, Z, W)=(11,31,29,1) \\
=\left(\begin{array}{c}
264009744251,722953698209, \\
676722505229,70657231681
\end{array}\right) \\
(m, n)=(3,2): 12 X^{4}+5 Y^{4}=12 Z^{4}+5 W^{4} \\
(m, n)=(4,1): 8 X^{4}+15 Y^{4}=8 Z^{4}+15 W^{4}
\end{gathered}
$$

\section{Case (B):}

We show Diophantine Equation:

$$
\left(m^{4}+1\right) X^{4}+\left(m^{4}+1\right) Y^{4}=2 Z^{4}+2 W^{4}
$$


has infinitely many integer solutions.

$m$ is arbitrary.

Since in (1a) above, $(\mathrm{a}, \mathrm{b}, \mathrm{c}, \mathrm{d})=\left[\left(m^{4}+1\right),\left(m^{4}+1\right),(2)(2)\right]$ the product $($ abcd $)$ becomes a square $=\left[2\left(m^{4}+1\right)\right]$. If ' $\mathrm{m}$ ' is written as $(\mathrm{p} / \mathrm{q})$ the above can be re-written as below:

$$
\left(m^{4}+n^{4}\right) X^{4}+\left(m^{4}+n^{4}\right) Y^{4}=2 Z^{4}+2 W^{4} .
$$

$\mathrm{m}, \mathrm{n}$ are arbitrary.

$$
\begin{gathered}
\left(m^{4}+1\right) X^{4}+\left(m^{4}+1\right) Y^{4}=2 Z^{4}+2 W^{4} \\
\text { We use an identity } p(t+1)^{4}+p(t)^{4} \\
=\left(t^{2}+a t+b\right)^{2}+\left(c t^{2}+d t+e\right)^{2}, \\
\text { with }(a, b, c, e, p)=\left(d+2, \frac{d}{2}+1,1+d, \frac{d}{2}, 1+d+\frac{d^{2}}{2}\right) \\
\text { So, we look for the integer solutions }\left\{\begin{array}{c}
Z^{2}=t^{2}+(d+2) t+\frac{d}{2}+1, \\
W^{2}=(1+d) t^{2}+d t+\frac{d}{2}
\end{array}\right\}
\end{gathered}
$$

By parameterizing the first Equation and substituting the result to second Equation, then we obtain quartic Equation below to make ( $\mathrm{w}=$ square $)$.

Since, $(\mathrm{t}, \mathrm{z})=\left(-\frac{1}{2}, \frac{1}{2}\right)$ we get $(\mathrm{z}, w)=\left(\frac{1}{2}, \frac{m}{2}\right)$ so we parameterize for $(\mathrm{t}, \mathrm{z})$

We get:

$$
t=-\frac{\left(k^{2}+2 k-2 d-3\right)}{2\left(k^{2}-1\right)}
$$

Hence we get:

$$
v^{2}=(d+1) k^{4}+4 k^{3}-2 k^{2}(d+1)-\left(12+8 d^{2}+16 d\right)+\left(9+17 d+4 d^{3}+12 d^{2}\right)
$$

In-order to make the coefficient of $(k)^{4}$ a square we take $\left(d+1=m^{2}\right)$

$$
\begin{aligned}
& \text { Hence let } d=m^{2}-1 \text {, then substituting }\left(d=m^{2}-1\right) \text { in }(3 a) \text { we get } \\
& v^{2}=k^{4} m^{2}+4 k^{3}-2 k^{2} m^{2}+\left(-8 m^{4}-4\right) k+\left(4 m^{6}+5 m^{2}\right)
\end{aligned}
$$

This quartic Equation (4) is bi-rationally equivalent to an elliptic curve below.

$$
\begin{gathered}
Y^{2}-\frac{4 Y X}{m}+\left(8 m+16 m^{5}\right) Y=X^{3}-\frac{2\left(m^{4}+2\right) X^{2}}{m^{2}}+\left(-20 m^{4}-16 m^{8}\right) X+104 m^{6}+32 m^{10}+80 m^{2} \\
\text { It has a point } P(X, Y)=\left(\frac{2\left(m^{4}+2\right)}{m^{2}},-\frac{16\left(-1+m^{8}\right)}{m^{3}}\right) . \\
\text { Hence we get } 2 P(X, Y)=\left(\frac{m^{8}+4 m^{4}+1}{m^{2}},-\frac{3 m^{8}-m^{4}-3+m^{12}}{m^{3}}\right) .
\end{gathered}
$$

This point $\mathrm{P}$ is of infinite order, and the multiples " $\mathrm{nP}$ ", $\mathrm{n}=2,3, \ldots$ give infinitely many points.

This quartic Equation (4) above has infinitely many parametric solutions, one of which is shown below.

$$
\begin{gathered}
n=2: \\
X=m^{4}+4 m^{2}-1 \\
Y=m^{4}-4 m^{2}-1 \\
Z=3 m^{4}+1 \\
W=\left(m^{4}+3\right) m \\
\mathrm{n}=3 \\
X=\left(m^{12}+12 m^{10}-19 m^{8}+40 m^{6}+19 m^{4}+12 m^{2}-1\right) \\
Y=\left(m^{12}-12 m^{10}-19 m^{8}-40 m^{6}+19 m^{4}-12 m^{2}-1\right)
\end{gathered}
$$




$$
\begin{aligned}
Z & =\left(5 m^{12}-27 m^{8}-41 m^{4}-1\right) \\
W & =m\left(m^{12}+41 m^{8}+27 m^{4}-5\right)
\end{aligned}
$$

etc.

Hence we can obtain infinitely many integer solutions for Equation (1).

Example: $m=2$,

$$
\begin{gathered}
17 X^{4}+17 Y^{4}=2 Z^{4}+2 W^{4}: \\
{[X, Y, Z, W]=[31,1,49,38],} \\
{[14431,15361,12911,30038], \ldots \text { etc } .}
\end{gathered}
$$

\section{Case (C):}

$$
\left(d^{2}-2 d m^{2}+2 m^{4}\right) X^{4}+\left(d^{2}-2 d m^{2}+2 m^{4}\right) Y^{4}=2\left(-d+m^{2}\right)^{2} Z^{4}+2 W^{4}
$$

We show Diophantine Equation,

$$
\left(d^{2}-2 d m^{2}+2 m^{4}\right) X^{4}+\left(d^{2}-2 d m^{2}+2 m^{4}\right) Y^{4}=2\left(-d+m^{2}\right)^{2} Z^{4}+2 W^{4}
$$

has infinitely many integer solutions.

$\mathrm{d}, \mathrm{m}$ are arbitrary.

$$
\left(d^{2}-2 d m^{2}+2 m^{4}\right) X^{4}+\left(d^{2}-2 d m^{2}+2 m^{4}\right) Y^{4}=2\left(-d+m^{2}\right)^{2} Z^{4}+2 W^{4}
$$

We use an identity

$$
\begin{gathered}
p(t+1)^{4}+p(t)^{4}=q\left(t^{2}+a t+b\right)^{2}+\left(c t^{2}+d t+e\right)^{2} \\
\text { with }(a, b, e, p, q)=\frac{d-2 c}{d-c}, \frac{(d-2 c)}{2(d-c)}, \frac{d}{2}, \frac{d^{2}-2 d c+2 c^{2}}{2},(c-d)^{2}
\end{gathered}
$$

So, we look for the integer solutions

$$
\left\{\begin{array}{l}
Z^{2}=t^{2}+\frac{(d-2 c) t}{(d-c)}+\frac{d-2 c}{2(d-c)}, \\
W^{2}=c t^{2}+d t+\frac{d}{2}
\end{array}\right\}
$$

By parameterizing the first Equation and substituting the result to second Equation, then we obtain quartic Equation (4) below.

$$
\begin{gathered}
\text { Let } c=m^{2}, \text { then } \\
u^{2}=m^{2}\left(-d+m^{2}\right)^{2} k^{4}+\left(4 m^{6}-12 d m^{4}+12 m^{2} d^{2}-4 d^{3}\right) k^{3}+\left(4 d m^{4}-2 m^{2} d^{2}-2 m^{6}\right) k^{2}+\left(-12 m^{6}+\right. \\
\left.20 d m^{4}-12 m^{2} d^{2}+4 d^{3}\right) k+5 m^{2} d^{2}+9 m^{6}-10 d m^{4}
\end{gathered}
$$

This quartic Equation (4) is bi-rationally equivalent to an elliptic curve below.

$$
\begin{aligned}
& Y^{2}-4\left(-2 d 8 m^{2}+d^{2}+m^{4}\right) \frac{X Y}{m}+\left(24 m^{9}-64 d m^{7}+64 m^{5} d^{2}-32 m^{3} d^{3}+8 m d^{4}\right) Y \\
& =X^{3}-\frac{2\left(2 d^{4}-8 m^{2} d^{3}+13 m^{4} d^{2}-10 d m^{6}+3 m^{8}\right) X^{2}}{m^{2}} \\
& +\left(80 m^{6} d^{3}-20 m^{4} d^{4}-136 m^{8} d^{2}+112 m^{10} d-36 m^{12}\right) X+80 m^{2} d^{8}-640 d^{7} m^{4}+2344 d^{6} m^{6} \\
& -5104 d^{5} m^{8}+7192 d^{4} m^{10}-6688 d^{3} m^{12}+3992 d^{2} m^{14}-1392 d m^{16}+216 m^{18} \\
& \text { It has a point } P(X, Y)=\left(2\left(2 d^{4}-8 m^{2} d^{3}+13 m^{4} d^{2}-10 d m^{6}+3 m^{8}\right) \frac{1}{m^{2}}\right. \\
& \left.16\left(d^{5}-6 m^{2} d^{4}+15 m^{4} d^{3}-20 m^{6} d^{2}+14 m^{8} d-4 m^{10}\right) \frac{d}{m^{3}}\right) \\
& \text { Hence we get } 2 P(X, Y)=\left(\begin{array}{c}
\frac{d^{4}-4 m^{2} d^{3}+10 m^{4} d^{2}-12 d m^{6}+6 m^{8}}{m^{2}}, \\
\frac{d\left(3 d^{5}-18 m^{2} d^{4}+46 m^{4} d^{3}-64 m^{6} d^{2}+48 m^{8} d-16 m^{10}\right)}{m^{3}}
\end{array}\right)
\end{aligned}
$$


This point $\mathrm{P}$ is of infinite order, and the multiples $\mathrm{nP}, \mathrm{n}=2,3, \ldots$ give infinitely many points. This quartic Equation has infinitely many parametric solutions below.

$$
\begin{gathered}
n=2: \\
X=4 m^{4}-2 d m^{2}-d^{2} \\
Y=4 m^{4}-6 d m^{2}+d^{2} \\
Z=4 m^{4}-2 d m^{2}+d^{2} \\
W=m\left(4 m^{4}-6 d m^{2}+3 d^{2}\right) \\
n=3: \\
X=64 m^{12}-224 m^{10} d+320 m^{8} d^{2}-216 m^{6} d^{3}+64 m^{4} d^{4}-6 d^{5} m^{2}-d^{6} \\
Y=64 m^{12}-160 m^{10} d+160 m^{8} d^{2}-104 m^{6} d^{3}+56 m^{4} d^{4}-18 d^{5} m^{2}+d^{6} \\
Z=64 m^{12}-224 d m^{10}+288 d^{2} m^{8}-184 d^{3} m^{6}+56 d^{4} m^{4}-6 d^{5} m^{2}+d^{6} \\
W=m\left(64 m^{12}-160 d m^{10}+128 d^{2} m^{8}-8 d^{3} m^{6}-48 d^{4} m^{4}+30 d^{5} m^{2}-5 d^{6}\right)
\end{gathered}
$$

etc.

Hence we can obtain infinitely many integer solutions for Equation (1).

Example:

$$
\begin{gathered}
(d, m)=(2,2): \quad 10 X^{4}+10 Y^{4}=4 Z^{4}+W^{4}: \\
{[X, Y, Z, W]=[11,5,13,14],[563,893,277,1646], \ldots \text { etc } .}
\end{gathered}
$$

\section{Case (D)}

$$
d m^{2}(-d-2) X^{4}+\left(-2 m^{2}+d+2\right) m^{2}\left(d-2 m^{2}\right) Y^{4}=m^{4} d\left(d-2 m^{2}\right) Z^{4}+(d+2)\left(2 m^{2}-d-2\right) W^{4}
$$

We show above Diophantine Equation has infinitely many integer solutions. $d, m$ are arbitrary.

$$
d m^{2}(-d-2) X^{4}+\left(-2 m^{2}+d+2\right) m^{2}\left(d-2 m^{2}\right) Y^{4}=m^{4} d\left(d-2 m^{2}\right) Z^{4}+(d+2)\left(2 m^{2}-d-2\right) W^{4}
$$

We use an identity,

$$
\begin{gathered}
p(t+1)^{4}+q(t)^{4}=r\left(t^{2}+a t+b\right)^{2}+\frac{p q\left(c t^{2}+d t+e\right)^{2}}{r} \\
\text { with }(a, b, e, p, q)=\left(\frac{d+2}{c}, \frac{d+2}{2 c}, \frac{d}{2}, \frac{r(d+2)}{c(-d+2 c)},-\frac{(2 c-d-2) r}{d c}\right)
\end{gathered}
$$

So, we look for the integer solutions

$$
\begin{gathered}
\left\{Z^{2}=\left(t^{2}+\frac{(d+2) t}{c}+\frac{d+2}{2 c}\right),\right. \\
\left.W^{2}=\left(c t^{2}+d t+\frac{d}{2}\right)\right\}
\end{gathered}
$$

By parameterizing the first Equation and substituting the result to second Equation, then we obtain quartic Equation below.

$$
\begin{gathered}
\text { Let } c=m^{2}, \text { then } \\
u^{2}=m^{4} k^{4}+\left(-4 m^{2} d+4 m^{4}\right) k^{3}+\left(4 d^{2}-8 m^{2} d+6 m^{4}-8 m^{2}+8 d\right) k^{2}+\left(-16 m^{2}+4 m^{4}-4 m^{2} d\right) k+16+ \\
m^{4}-8 m^{2}+8 d
\end{gathered}
$$

This quartic Equation is bi-rationally equivalent to an elliptic curve below.

$$
\begin{gathered}
Y^{2}+\left(-4 d+4 m^{2}\right) Y X+\left(-32 m^{4}+8 m^{6}-8 m^{4} d\right) Y \\
=X^{3}+\left(2 m^{4}-8 m^{2}+8 d\right) X^{2}+\left(-64 m^{4}-4 m^{8}+32 m^{6}-32 m^{4} d\right) X-384 m^{8}-8 m^{12} \\
+96 m^{10}-96 m^{8} d+512 m^{6}+512 m^{6} d-512 m^{4} d-256 m^{4} d^{2} \\
\text { It has a point } P(X, Y)=\left(-2 m^{4}+8 m^{2}-8 d, 64 m^{2} d-32 d^{2}\right) . \\
\text { Hence we get } 2 P(X, Y)=\left(4+2 m^{4}, 8 m^{4}+8+16 d-16 m^{2}\right) .
\end{gathered}
$$


This point $\mathrm{P}$ is of infinite order, and the multiples $\mathrm{nP}, \mathrm{n}=2,3, \ldots$ give infinitely many points.

This quartic Equation has infinitely many parametric solutions below.

$$
\begin{gathered}
X=3 m^{4}+(-2-2 d) m^{2}-1 \\
Y=m^{4}+(-2-2 d) m^{2}+1 \\
Z=m^{4}+2 m^{2}-2 d-3 \\
W=m\left(m^{4}-2 m^{2}+2 d+1\right) \\
n=3: \\
X=5 m^{12}+(-10-10 d) m^{10}+\left(8 d-9+4 d^{2}\right) m^{8}+(36+36 d) m^{6}+\left(-28 d^{2}-56 d-29\right) m^{4} \\
+\left(24 d^{2}+8 d^{3}+6+22 d\right) m^{2}+1 \\
Y=m^{12}+(-6-6 d) m^{10}+\left(8 d+4 d^{2}+15\right) m^{8}+(-20-20 d) m^{6}+\left(40 d+20 d^{2}+15\right) m^{4} \\
+\left(-24 d^{2}-8 d^{3}-6-22 d\right) m^{2}+1
\end{gathered}
$$

etc.

Hence we can obtain infinitely many integer solutions for Equation (1).

Example:

$$
\begin{gathered}
(d, m)=(2,2): \\
-2 X^{4}+6 Y^{4}=-12 Z^{4}+W^{4}
\end{gathered}
$$

Or

$$
\begin{gathered}
12 Z^{4}+6 Y^{4}=2 X^{4}+W^{4} \\
{[X, Y, Z, W]=[23,7,17,26]} \\
{[647,4183,2993,7274], \ldots \text { etc. }}
\end{gathered}
$$

Equation of the type:

$$
p a^{m}+q b^{m}+r c^{n}+s d^{n}=0
$$

where: $(m, n)=(2,4)$

Note: We have attempted to parameterize a similar family of Equation related to Equation (1) at the top of the paper. Difference being Equation above is mixed powers of degree four \& two. Others are free to attempt to make the two square components a fourth power. Thus making Equation (2) eligible for the Equation (1) group.

\section{Case A:}

$$
p a^{2}+q b^{2}+r c^{4}+s d^{4}=0
$$

Let,

$$
(p, q, r, s)=(1,1,-1,-1)
$$

Substitution above values in Equation (1) we get:

$$
a^{2}+b^{2}=c^{4}+d^{4}
$$

Above is equivalent to:

$$
\left(a+c^{2}\right)\left(a-c^{2}\right)=\left(d^{2}+b\right)\left(d^{2}-b\right)
$$

Since, $(p q)(r s)=(p s)(r q)$, let:

$$
\begin{aligned}
& \left(a+c^{2}\right)=p q \\
& \left(a-c^{2}\right)=r s
\end{aligned}
$$




$$
\begin{aligned}
& \left(d^{2}+b\right)=p s \\
& \left(d^{2}-b\right)=r q
\end{aligned}
$$

Hence we get:

$$
\begin{gathered}
2 c^{2}=p q-r s \\
2 d^{2}=p s+q r \\
2 a=p q+r s \\
2 b=p s-q r \\
\text { Let, } q=2(p+r) \quad \& \quad s=2 p
\end{gathered}
$$

Therefore we get: $c=p, \&$

$$
d^{2}=\left(p^{2}+p r+r^{2}\right)
$$

Above Equation (2) is parameterized as:

$$
(p, r, d)=\left[\left(m^{2}-n^{2}\right),\left(2 m n+n^{2}\right),\left(m^{2}+m n+n^{2}\right)\right]
$$

Hence we get: $(c, d)=\left[\left(m^{2}-n^{2}\right),\left(m^{2}+m n+n^{2}\right)\right]$

Since, $2 a=p q+r s$

we get, $a=\left(m^{2}-n^{2}\right)\left(m^{2}+4 m n+n^{2}\right)$

And as,

$$
2 b=p s-q r
$$

we get, $b=\left(m^{4}-2 m^{3} n-7 m^{2} n^{2}-2 m n^{3}+n^{4}\right)$

Summarizing, $a=\left(m^{2}-n^{2}\right)\left(m^{2}+4 m n+n^{2}\right)$

$$
\begin{gathered}
b=\left(m^{4}-2 m^{3} n-7 m^{2} n^{2}-2 m n^{3}+n^{4}\right) \\
c=\left(m^{2}-n^{2}\right) \\
d=\left(m^{2}+m n+n^{2}\right)
\end{gathered}
$$

for, $(m, n)=(3,2)$

$$
(a, b, c, d)=(185,311,5,19)
$$

\& we get the numerical solution:

$$
\left(5^{4}+19^{4}\right)=\left(185^{2}+311^{2}\right)
$$

Additional Example:

\section{Case B:}

$$
\begin{gathered}
p a^{m}+q b^{m}+r c^{n}+s d^{n}=0 \\
(p, q, r, s)=(5,-5,1,-1) \\
(m, n)=(2,4)
\end{gathered}
$$

Since we have,

$$
5 a^{2}+c^{4}=5 b^{2}+d^{4}
$$

Hence, $5\left(b^{2}-a^{2}\right)=c^{4}-d^{4}$

$$
5(b+a)(b-a)=\left(c^{2}+d^{2}\right)\left(c^{2}-d^{2}\right),
$$

Therefore we take,

$$
\begin{gathered}
c^{2}-d^{2}=(b+a) \\
c^{2}+d^{2}=5(b-a)
\end{gathered}
$$

Thus we have,

$$
c^{2}=(3 b-2 a) \& d^{2}=(2 b-3 a)
$$

Taking, $a=\left(10 m^{2}-15 n^{2}\right) \& b=\left(15 m^{2}-10 n^{2}\right)$ 
we get, $(c, d)=(5 m, 5 n)$

For, $(m, n)=(3,2)$ we get,

$$
\begin{gathered}
(a, b, c, d)=(30,95,15,10) \\
15^{4}+5(30)^{2}=10^{4}+5(95)^{2}
\end{gathered}
$$

Note: Reader's may be interested in the table of result's below. These were arrived at by elliptical method. We have not attempted to parameterize the family of Equations below, but others can make an attempt. Since knowing the numerical solution is helpful towards parameterization.

Table 1. (below for numerical solutions):

\begin{tabular}{|c|c|c|c|c|}
\hline $\begin{array}{c}\text { Equation type: } a x^{4}+b y^{4}=c z^{4}+d w^{4} \\
\text { product (abcd) }=\square(\text { square), } \\
\text { \& Condition, (a+b)=(c+16d) }\end{array}$ & $\mathrm{x}$ & $\mathrm{Y}$ & $\mathrm{Z}$ & $\mathrm{w}$ \\
\hline$x^{4}+20 y^{4}=5 z^{4}+w^{4}$ & 19 & 5 & 13 & 2 \\
\hline$x^{4}+27 y^{4}=12 z^{4}+w^{4}$ & 23 & 9 & 1 & 26 \\
\hline$x^{4}+49 y^{4}=18 z^{4}+2 w^{4}$ & 73 & 23 & 39 & 22 \\
\hline $2 x^{4}+18 y^{4}=4 z^{4}+w^{4}$ & 13 & 3 & 11 & 2 \\
\hline $2 x^{4}+28 y^{4}=14 z^{4}+w^{4}$ & 17 & 7 & 1 & 22 \\
\hline $3 x^{4}+25 y^{4}=12 z^{4}+w^{4}$ & 9 & 131 & 157 & 92 \\
\hline $3 x^{4}+49 y^{4}=4 z^{4}+3 w^{4}$ & 1 & 31 & 41 & 58 \\
\hline $5 x^{4}+20 y^{4}=9 z^{4}+w^{4}$ & 17 & 7 & 15 & 10 \\
\hline
\end{tabular}

\section{Section (2)}

$$
a x^{4}+b y^{4}+c z^{4}+d w^{4}=0
$$

[Condition: Product of coefficient's (abcd) not equal to a square]

\section{Case (A)}

$$
p X^{4}+p Y^{4}+2 Z^{4}=s W^{4}
$$

Introduction:

Existence of solution for Diophantine Equation

$$
a x^{4}+b y^{4}+c z^{4}+d w^{4}=0
$$

are known if (abcd) is square number.

So, we are curious about whether above Equation has a solution or not if abcd is not a square number. In particular, when does this Equation have infinitely many integer solutions Since Richmond (ref \# 2) conjectured that, if there is one rational solution there are infinite more.

We show Diophantine Equation: $p x^{4}+q y^{4}+r z^{4}=s w^{4}$

For $(\mathrm{p}, \mathrm{q}, \mathrm{r}, \mathrm{s})=(7,7,1,8)$

$$
7 X^{4}+7 Y^{4}+Z^{4}=8 W^{4}
$$

has infinitely many integer solutions (numerical method).

Multiplying throughout by two the above Equation we get:

$$
14 X^{4}+14 Y^{4}+2 Z^{4}=W^{4}
$$

Which is a (4-1-30) quartic Equation:

$$
7 X^{4}+7 Y^{4}+Z^{4}=8 W^{4}
$$

We use an identity

$$
7(t+4)^{4}+7(2 t)^{4}+\left(3 t^{2}+24 t-16\right)^{2}=8\left(4\left(t^{2}+t+4\right)\right)^{2}
$$




$$
\text { So, we look for the integer solutions }\left\{\begin{array}{l}
Z^{2}=3 t^{2}+24 t-16, \\
W^{2}=4 t^{2}+4 t+16
\end{array}\right\}
$$

By parameterizing the second Equation and substituting the result to first Equation, then we obtain quartic Equation below.

$$
u^{2}=k^{4}+12 k^{3}-26 k^{2}-36 k+37
$$

This quartic Equation is bi-rationally equivalent to an elliptic curve below.

$$
y^{2}=x^{3}+x^{2}-50 x+48 .
$$

Rank is 1 and generator is $[-2,-12]$.

Hence we can obtain infinitely many integer solutions for Equation (1).

Numerical example:

$$
[X, Y, Z, W]=[48,5,37,47],[11395,83472,87023,104413]
$$

From above we deduce:

$$
\begin{gathered}
W^{4}=14 X^{4}+14 Y^{4}+2 Z^{4} \\
\text { Let : } p x^{4}+q y^{4}+r z^{4}=w^{4}
\end{gathered}
$$$$
\text { For }(\mathrm{p}, \mathrm{q}, \mathrm{r})=(14,14,2)
$$$$
(X, Y, Z, W)=(48,5,37,94)
$$

Let: $\mathrm{n}=(\mathrm{p}+\mathrm{q}+\mathrm{r})$

Above Equation (1) is a (4-1-n) quartic Equation. For $n=(14+14+2)=30$ which we write as $(4-1-n)=(4-1-30)$ Equation.

There are more (4-1-n) numerical solution's as derived by elliptical method for $(n<50) \&$ is shown below:

$$
\begin{gathered}
(94)^{4}=14(48)^{4}+14(5)^{4}+2(37)^{4}-----(4-1-30) \text { eqn. } \\
(7)^{4}=9(4)^{4}+(1)^{4}+6(2)^{4}-------(4-1-16) \text { eqn. } \\
(721)^{4}=36(200)^{4}+(529)^{4}+3(460)---(4-1-40) \text { eqn. } \\
(9)^{4}=25(4)^{4}+(1)^{4}+10(2)^{4}------(4-1-36) \text { eqn. } \\
(32)^{4}=12(17)^{4}+3(11)^{4}+(7)^{4}------(4-1-16) \text { eqn. }
\end{gathered}
$$
12).

Note: For more (4-1-n) Equation numerical solutions interested reader can see table (3) at the end of this paper, (ref \#

Parametric method in-lieu of the above elliptical numerical method (eqn. (4):

We show Diophantine Equation $p X^{4}+p Y^{4}+2 Z^{4}=s W^{4}$ has infinitely many integer solutions.

$$
\begin{gathered}
p=a^{2}-2, s=a^{2} \\
p X^{4}+p Y^{4}+2 Z^{4}=s W^{4}
\end{gathered}
$$

We use an identity:

$$
\begin{gathered}
\left.\qquad \begin{array}{c}
p(a t+a)^{4}+p(t)^{4}+r\left(t^{2}+a^{2} t+a^{2}\right)^{2}=s\left(c t^{2}+2 c t+a^{2}\right)^{2} \\
\text { with, } s=p+r, \quad r=\frac{2 p}{a^{2}-2}, \quad c=a^{2}-1 . \\
\text { So, we look for the integer solutions }\left\{\begin{array}{c}
Z^{2}=t^{2}+a^{2} t+a^{2}, \\
W^{2}=
\end{array} \quad\left(a^{2}-1\right) t^{2}+2\left(a^{2}-1\right) t+a^{2}\right.
\end{array}\right\}
\end{gathered}
$$

By parameterizing the second Equation and substituting the result to first Equation, then we obtain quartic Equation below.

$$
u^{2}=k^{4}+\left(4 a^{2}-6\right) k^{2}-\left(+4 a^{4}+8-12 a^{2}\right) k+\left(-3+8 a^{2}-5 a^{4}+a^{6}\right)
$$

This quartic Equation is bi-rationally equivalent to an elliptic curve below.

$$
\begin{gathered}
Y^{2}+\left(-8 a^{4}-16+24 a^{2}\right) Y \\
=X^{3}+\left(4 a^{2}-6\right) X^{2}+\left(12-32 a^{2}+20 a^{4}-4 a^{6}\right) X+\left(240 a^{2}-248 a^{4}+104 a^{6}-16 a^{8}-72\right) .
\end{gathered}
$$

The corresponding point is $P(X, Y)=\left(6-4 a^{2}, 8 a^{4}-24 a^{2}+16\right)$. 
Hence we get $2 \mathrm{P}(\mathrm{X}, \mathrm{Y})$,

$$
\begin{gathered}
X=\frac{a^{4}+4 a^{2}+12}{4}, \\
Y=\frac{a^{6}+14 a^{4}-132 a^{2}+72}{8}
\end{gathered}
$$

This point $\mathrm{P}$ is of infinite order, and the multiples $\mathrm{mP}, \mathrm{m}=2,3, \ldots$ give infinitely many points.

This quartic Equation has infinitely many parametric solutions below.

$$
\begin{gathered}
p=\left(-2+a^{2}\right), \quad s=a^{2} \\
m=2: \\
X=8 a\left(a^{2}+2\right) \\
Y=\left(a^{2}-4 a-2\right)\left(a^{2}+4 a-2\right) \\
Z=\left(-4-20 a^{2}+3 a^{4}\right) \\
W=\left(a^{4}+20 a^{2}-12\right) \\
m=3: \\
X=8 a\left(a^{4}+20 a^{2}-12\right)^{2}\left(a^{2}+2\right)\left(-4-20 a^{2}+3 a^{4}\right) \\
Y=\left(a^{4}+20 a^{2}-12\right)\left(a^{2}-4 a-2\right)\left(a^{2}+4 a-2\right) * \\
\left(a^{4}-8 a^{3}-20 a^{2}-16 a+4\right) *\left(a^{4}+8 a^{3}-20 a^{2}+16 a+4\right) \\
Z=\left(a^{4}+20 a^{2}-12\right) * \\
\left(64+3008 a^{2}-6096 a^{4}+9120 a^{6}-932 a^{8}-228 a^{10}+5 a^{12}\right) \\
W=\left(a^{4}+20 a^{2}-12\right) * \\
\left(a^{12}+188 a^{10}-1524 a^{8}+9120 a^{6}-3728 a^{4}-3648 a^{2}+320\right)
\end{gathered}
$$

etc.

Hence we can obtain infinitely many integer solutions for Equation (1).

Example:

$$
a=4: \quad 7 X^{4}+7 Y^{4}+Z^{4}=8 W^{4}
$$

Numerical example:

$$
\begin{gathered}
\text { We take }(a, m)=(4,2) \\
{[X, Y, Z, W]=[48,5,37,47]}
\end{gathered}
$$

From above we deduce:

$$
\begin{gathered}
W^{4}=14 X^{4}+14 Y^{4}+2 Z^{4}----(4-1-30) \\
\text { quartic numerical equation given below. } \\
(94)^{4}=14(48)^{4}+14(5)^{4}+2(37)^{4}
\end{gathered}
$$

\section{Case (B):}

$$
p X^{4}+p Y^{4}=2 Z^{4}+W^{4}
$$

We show Diophantine Equation $\left(p X^{4}+p Y^{4}=2 Z^{4}+W^{4}\right)$ has infinitely many integer solutions for $p=1+2 b^{4}$. $\mathrm{b}$ is arbitrary.

$$
p\left(X^{4}+Y^{4}\right)=2 Z^{4}+W^{4} .
$$

We use an identity

$$
p(t+1)^{4}+p(t)^{4}=2\left(t^{2}+a t+b^{2}\right)^{2}+\left(c t^{2}+d t+e\right)^{2},
$$

$$
\text { with } p=1+2 b^{4}, a=2 b^{2}+1, c=-2 b^{2}, d=2-2 b^{2}, e=1 \text {. }
$$


So, we look for the integer solutions,

$$
\left\{Z^{2}=t^{2}+\left(2 b^{2}+1\right) t+b^{2}, W^{2}=-2 b^{2} t^{2}+\left(2-2 b^{2}\right) t+1\right\}
$$

By parameterizing the second Equation and substituting the result to first Equation, then we obtain quartic Equation below.

$$
u^{2}=k^{4}+\left(-4 b+4 b^{3}\right) k^{3}+\left(-12 b^{4}+2 b^{2}\right) k^{2}+\left(4 b^{3}+4 b+16 b^{5}\right) k-\left(4 b^{4}+4 b^{2}+8 b^{6}+1\right)
$$

This quartic Equation is bi-rationally equivalent to an elliptic curve below.

$$
\begin{gathered}
Y^{2}+\left(-4 b+4 b^{3}\right) Y X+\left(8 b^{3}+8 b+32 b^{5}\right) Y=X^{3}+\left(-4 b^{4}-2 b^{2}-4 b^{6}\right) X^{2}+\left(16 b^{4}+16 b^{2}+32 b^{6}+4\right) X \\
-\left(192 b^{8}+112 b^{6}+192 b^{10}+48 b^{4}+8 b^{2}+128 b^{12}\right)
\end{gathered}
$$

The corresponding point is $P(X, Y)=\left(\left(4 b^{4}+2 b^{2}+4 b^{6}\right),\left(-24 b^{5}-16 b^{9}-8 b\right)\right)$.

$$
\begin{gathered}
\text { Hence we get } 2 P(X, Y)=\left(\frac{4 b^{8}+16 b^{6}-12 b^{4}+1}{4\left(b^{2}\right)},\right. \\
\left.(-) \frac{\left(52 b^{8}+80 b^{6}+18 b^{4}+24 b^{12}+48 b^{10}-4 b^{2}-1\right)}{8\left(b^{3}\right)}\right)
\end{gathered}
$$

This point $\mathrm{P}$ is of infinite order, and the multiples $\mathrm{mP}, \mathrm{m}=2,3, \ldots$ give infinitely many points.

This quartic Equation has infinitely many parametric solutions below.

$$
\begin{gathered}
p=2 b^{4}+1 . \\
b \text { is arbitrary. } \\
m=2: \\
X=\left(4 b^{8}+20 b^{4}+1\right) \\
Y=8\left(2 b^{4}-1\right) b^{2} \\
Z=b\left(-3-20 b^{4}+4 b^{8}\right) \\
W=\left(12 b^{8}+20 b^{4}-1\right) \\
m=\left(4 b^{8}+20 b^{4}+1\right)\left(16 b^{16}+416 b^{12}+152 b^{8}+104 b^{4}+1\right) \\
Y=8\left(2 b^{4}-1\right)\left(4 b^{8}-20 b^{4}-3\right) b^{2}\left(12 b^{8}+20 b^{4}-1\right) \\
Z=\left(64 b^{24}-3008 b^{20}-6096 b^{16}-9120 b^{12}-932 b^{8}+228 b^{4}+5\right) b \\
W=\left(1-188 b^{4}-1524 b^{8}-9120 b^{12}-3728 b^{16}+3648 b^{20}+320 b^{24}\right)
\end{gathered}
$$

etc.

Hence we can obtain infinitely many integer solutions for Equation (1).

Example:

$$
\begin{array}{cc}
b=1: & 3 X^{4}+3 Y^{4}=2 Z^{4}+W^{4} \\
b=2: & 33 X^{4}+33 Y^{4}=2 Z^{4}+W^{4}
\end{array}
$$

\section{Case (C)}

$$
(d-2)(d+2)^{2} X^{4}+(4 d-8) Y^{4}+Z^{4}=(4 d+8) W^{4}
$$

We show Diophantine Equation, $(d-2)(d+2)^{2} X^{4}+(4 d-8) Y^{4}+Z^{4}=(4 d+8) W^{4}$ has infinitely many integer solutions.

$\mathrm{d}$ is arbitrary.

$$
(d-2)(d+2)^{2} X^{4}+(4 d-8) Y^{4}+Z^{4}=(4 d+8) W^{4}
$$

We use an identity, $p((t+1))^{4}+q(t)^{4}=r\left(t^{2}+a t+a\right)^{2}+s\left(c t^{2}+d t+a\right)^{2}$, 


$$
\text { with }(a, c, p, q, r)=\left(1+\frac{d}{2}, \frac{d}{2}, \frac{s\left(-4+d^{2}\right)}{4}, \frac{s(-2+d)}{2+d},-\frac{4 s}{2+d}\right) .
$$

So, we look for the integer solutions

$$
\left\{\begin{array}{l}
Z^{2}=t^{2}+\left(1+\frac{d}{2}\right) t+1+\frac{d}{2}, \\
W^{2}=\frac{d t^{2}}{2}+d t+1+\frac{d}{2}
\end{array}\right\}
$$

By parameterizing the second Equation and substituting the result to first Equation, then we obtain quartic Equation below.

$$
u^{2}=16 k^{4}+(-32+32 d) k^{2}+\left(-16 d^{2}+32 d\right) k+\left(-8 d^{2}+2 d^{3}+16+8 d\right)
$$

This quartic Equation is birationally equivalent to an elliptic curve below.

$$
\begin{gathered}
Y^{2}+\left(-128 d^{2}+256 d\right) Y=X^{3}+(-32+32 d) X^{2}+\left(512 d^{2}-128 d^{3}-1024-512 d\right) X \\
+\left(-32768 d^{2}+20480 d^{3}+32768-16384 d-4096 d^{4}\right)
\end{gathered}
$$

The corresponding point is $P(X, Y)=\left(32-32 d, 128 d^{2}-256 d\right)$.

Hence we get $2 P(X, Y)=\left(d^{2}+12 d+68,-404 d-360+34 d^{2}+d^{3}\right)$.

This point $\mathrm{P}$ is of infinite order, and the multiples $\mathrm{nP}, \mathrm{n}=2,3, \ldots$ give infinitely many points.

This quartic Equation has infinitely many parametric solutions below.

For $n=2$

$$
\begin{gathered}
X=16 d+96 \\
Y=-d^{2}+36 d+60 \\
Z=2\left(3 d^{2}-28 d-84\right) \\
W=d^{2}+44 d+36
\end{gathered}
$$

For $\mathrm{n}=3$,

$$
\begin{gathered}
X=16\left(d^{2}+44 d+36\right)(d+6)\left(3 d^{2}-28 d-84\right) \\
Y=\left(d^{2}-36 d-60\right)\left(d^{4}-200 d^{3}-616 d^{2}-3360 d-5616\right) \\
Z=2\left(316224+426816 d+215472 d^{2}+25696 d^{3}-7988 d^{4}-396 d^{5}+5 d^{6}\right) \\
W=\left(46656+355392 d+262128 d^{2}+39392 d^{3}-2276 d^{4}+388 d^{5}+d^{6}\right)
\end{gathered}
$$

etc.

Hence we can obtain infinitely many integer solutions for Equation (1).

Example:

$$
\begin{array}{cc}
d=1: & Z^{4}=12 W^{4}+9 X^{4}+4 Y^{4} \\
d=3: & 25 X^{4}+4 Y^{4}+Z^{4}=20 W^{4}
\end{array}
$$

Re: $\mathrm{d}=1$, Is equivalent to (4-1-25) Equation:

\section{Case (D)}

$$
\left(m^{2}+1\right)^{2}\left(m^{2}-1\right) X^{4}+\left(m^{2}-1\right) Y^{4}+\left(m^{2}+1\right) Z^{4}=2 m^{2}(W)^{4}
$$

We show Diophantine Equation above has infinitely many integer solutions.

' $\mathrm{m}$ ' is arbitrary.

$$
\left(m^{2}+1\right)^{2}\left(m^{2}-1\right) X^{4}+\left(m^{2}-1\right) Y^{4}+\left(m^{2}+1\right) Z^{4}=2 m^{2} W^{4}
$$

We use an identity:

$$
\begin{gathered}
p(t+1)^{4}+q(t)^{4}+r\left(t^{2}+a t+b\right)^{2}=s\left(c t^{2}+d t+e\right)^{2} \\
\text { with }(a, b, e, p, q, r)=\left(2, \frac{d}{-c+d}, d,-\frac{s d^{2}(d-2 c)}{2 c},-\frac{s c(d-2 c)}{2}, \frac{s d\left(d^{2}+c^{2}-2 c d\right)}{2 c}\right)
\end{gathered}
$$


So, we look for the integer solutions

$$
\left\{Z^{2}=\frac{\left(t^{2}+2 t+d\right)}{(-c+d)}, W^{2}=c t^{2}+d t+d\right\}
$$

By parameterizing the second Equation and substituting the result to first Equation, then we obtain quartic Equation below.

$$
\begin{gathered}
\operatorname{Let}(c, d)=\left(m^{2}, m^{2}+1\right), \text { then, } \\
u^{2}=m^{2} k^{4}+\left(-2 m^{4}+4 m^{2}\right) k^{2}+\left(4 m^{3}-4 m\right) k+\left(m^{6}+m^{4}-2 m^{2}+1\right)
\end{gathered}
$$

This quartic Equation is bi-rationally equivalent to an elliptic curve below.

$$
\begin{aligned}
Y^{2}+\left(8 m^{4}-8 m^{2}\right) Y= & X^{3}+\left(-2 m^{4}+4 m^{2}\right) X^{2}+\left(-4 m^{8}-4 m^{6}+8 m^{4}-4 m^{2}\right) X+ \\
& \left(8 m^{12}-8 m^{10}-32 m^{8}+40 m^{6}-16 m^{4}\right)
\end{aligned}
$$

The corresponding point is $\mathrm{P}(\mathrm{X}, \mathrm{Y})=\left(2 m^{4}-4 m^{2},-8 m^{4}+8 m^{2}\right)$.

$$
\text { Hence we get } 2 P(X, Y)=\left(\frac{1}{8}\left(34 m^{4}+12 m^{2}+2\right),-\frac{1}{8}\left(45 m^{6}+101 m^{4}-17 m^{2}-1\right)\right. \text {. }
$$

This point $\mathrm{P}$ is of infinite order, and the multiples $\mathrm{nP}, \mathrm{n}=2,3, \ldots$ give infinitely many points.

This quartic Equation has infinitely many parametric solutions below.

For $\mathrm{n}=2$,

$$
\begin{gathered}
X=8 m\left(3 m^{2}+1\right) \\
Y=15 m^{4}+18 m^{2}-1 \\
Z=9 m^{4}+22 m^{2}+1 \\
W=21 m^{4}+14 m^{2}-3
\end{gathered}
$$

For $\mathrm{n}=3$,

$$
\begin{gathered}
X=8\left(3 m^{2}+1\right)\left(-3+14 m^{2}+21 m^{4}\right) m\left(1+22 m^{2}+9 m^{4}\right) \\
Y=\left(15 m^{4}+18 m^{2}-1\right)\left(351 m^{8}+420 m^{6}+154 m^{4}+100 m^{2}-1\right) \\
Z=729 m^{12}+11106 m^{10}+16383 m^{8}+4924 m^{6}-569 m^{4}+194 m^{2}+1 \\
W=4941 m^{12}+13338 m^{10}+13467 m^{8}+3212 m^{6}-1997 m^{4}-198 m^{2}+5
\end{gathered}
$$

etc.

Hence we can obtain infinitely many integer solutions for Equation (1).

Example:

$$
\begin{array}{cc}
m=2: & 75 X^{4}+3 Y^{4}+5 Z^{4}=8 W^{4}: \\
m=3: & 25 X^{4}+4 Y^{4}+5 Z^{4}=9 W^{4}
\end{array}
$$

For $\mathrm{m}=2$, multiplying throughout by two we get:

$\left(S^{4}=75 P^{4}+3 Q^{4}+R^{4}\right)$ which is a (4-1-79) quartic Equation.

Example:

$$
p a^{m}+q b^{m}+r c^{n}+s d^{n}=0
$$

Note: We have attempted to parameterize a similar family of Equation related to Equation (1) at the top of the paper. Difference being Equation above is mixed powers of degree four \& two. Others are free to attempt to make the two square components a fourth power. Thus making Equation (2) below eligible for the Equation (1) group above.

$$
p a^{m}+q b^{m}+r c^{n}+s d^{n}=0
$$

(pqrs) not a square,

$$
\begin{gathered}
(p, q, r, s)=(6,1,-9,-1) \\
(m, n)=(2,4)
\end{gathered}
$$

Hence Equation (1) becomes: 


$$
6 a^{2}+b^{2}=9 c^{4}+d^{4}
$$

Equation (3) has solution:

$$
(a, b, c, d)=\left[\left(2 t^{2}+6 t+3\right),\left(t^{2}+t\right),((t+1),(t)]\right.
$$

For $\mathrm{t}=6$, we get: $(a, b, c, d)=(7,6,42,111)$

$6(42)^{2}+(111)^{2}=9(7)^{4}+(6)^{4}$ After removing common factor's we get:

$$
6(14)^{2}+(37)^{2}=9(2)^{4}+(7)^{4}
$$

Also see table no. 4 at the end of this paper for results arrived at by brute force for Equation $\left(a x^{4}+b y^{4}+c z^{4}+\right.$ $\left.d w^{4}=0\right)$. For $(\mathrm{abcd})$ not equal to a square $\& \mathrm{k}=(\mathrm{a}+\mathrm{b}+\mathrm{c}+\mathrm{d})$ for $(\mathrm{k}<30)$.

(See Table (2) below), for numerical solutions:

Note: Reader's may be interested in the table of result's below. These were arrived at by elliptical method. We have not attempted to parameterize the family of Equations below, but others can make an attempt. Since knowing the numerical

\begin{tabular}{|c|c|c|c|c|}
\hline Equation: (table 3a) & $\mathrm{X}$ & Y & $\mathrm{Z}$ & \\
\hline $5 X^{4}+45 Y^{4}+Z^{4}=6 W^{4}$ & 23 & 56 & 47 & 93 \\
\hline $2 X^{4}+8 Y^{4}+Z^{4}=9 W^{4}$ & 30 & 1 & 19 & 21 \\
\hline $31 X^{4}+31 Y^{4}+Z^{4}=2 W^{4}$ & 352 & 235 & 917 & 894 \\
\hline $25 X^{4}+4 Y^{4}+Z^{4}=5 W^{4}$ & 2 & 3 & 7 & 5 \\
\hline $79 X^{4}+79 Y^{4}+4 Z^{4}=2 W^{4}$ & 13 & 3 & 37 & 47 \\
\hline $57 X^{4}+57 Y^{4}=8 Z^{4}+2 W^{4}$ & 4 & 3 & 7 & 1 \\
\hline $18 X^{4}+8 Y^{4}+Z^{4}=27 W^{4}$ & 43 & 91 & 37 & 69 \\
\hline $73 X^{4}+73 Y^{4}+Z^{4}=2 W^{4}$ & 2361 & 5481 & 1758 & 13587 \\
\hline $75 X^{4}+3 Y^{4}+2 Z^{4}=5 W^{4}$ & 3 & 11 & 1 & 10 \\
\hline $33 X^{4}+33 Y^{4}=2 Z^{4}+W^{4}$ & 37 & 20 & 56 & 83 \\
\hline $3 X^{4}+3 Y^{4}=2 Z^{4}+W^{4}$ & 25 & 8 & 19 & 31 \\
\hline $9 X^{4}+4 Y^{4}+2 Z^{4}=6 W^{4}$ & 72 & 355 & 239 & 329 \\
\hline $25 X^{4}+25 Y^{4}+8 Z^{4}=25 W^{4}$ & 9 & 40 & 30 & 41 \\
\hline$X^{4}+9 Y^{4}+6 Z^{4}=W^{4}$ & 1 & 4 & 2 & 7 \\
\hline $9 X^{4}+36 Y^{4}+4 Z^{4}=9 W^{4}$ & 1 & 12 & 6 & 17 \\
\hline $7 X^{4}+7 Y^{4}+Z^{4}=8 W^{4}$ & 48 & 5 & 47 & 37 \\
\hline $47 X^{4}+47 Y^{4}+2 Z^{4}=49 W^{4}$ & 952 & 475 & 2073 & 1123 \\
\hline $7 X^{4}+7 Y^{4}+2 Z^{4}=9 W^{4}$ & 264 & 95 & 59 & 249 \\
\hline
\end{tabular}
solution is helpful towards parameterization.

\begin{tabular}{|c|c|c|c|c|}
\hline $\begin{array}{c}\text { Table }(2) \\
a X^{4}+b Y^{4}+c Z^{4}+d W^{4}=0\end{array}$ & $\mathrm{X}$ & $\mathrm{Z}$ & $\mathrm{W}$ \\
\hline $23 X^{4}+23 Y^{4}+2 Z^{4}=25 W^{4}$ & 360 & 43 & 457 & 371 \\
\hline$X^{4}+36 Y^{4}+27 Z^{4}=4 W^{4}$ & 51693 & 41613 & 38547 & 81294 \\
\hline $49 X^{4}+49 Y^{4}+8 Z^{4}=W^{4}$ & 3689 & 16892 & 17818 & 46819 \\
\hline $3 X^{4}+75 Y^{4}+5 Z^{4}=8 W^{4}$ & 22 & 15 & 17 & 28 \\
\hline $36 X^{4}+Y^{4}+3 Z^{4}=W^{4}$ & 200 & 529 & 460 & 721 \\
\hline $25 X^{4}+Y^{4}+10 Z^{4}=W^{4}$ & 4 & 1 & 2 & 9 \\
\hline $50 X^{4}+2 Y^{4}+5 Z^{4}=2 W^{4}$ & 72 & 1 & 12 & 161 \\
\hline $4 X^{4}+Y^{4}+Z^{4}=W^{4}$ & 72 & 49 & 84 & 113 \\
\hline $12 X^{4}+12 Y^{4}=8 Z^{4}+W^{4}$ & 9 & 17 & 19 & 14 \\
\hline $19 X^{4}+19 Y^{4}=2 Z^{4}+W^{4}$ & 8209 & 46228 & 81169 & 14397 \\
\hline $9 X^{4}+Y^{4}+2 Z^{4}=3 W^{4}$ & 40 & 47 & 37 & 57 \\
\hline$X^{4}+3 Y^{4}+Z^{4}=W^{4}$ & 17 & 11 & & 7 \\
\hline
\end{tabular}


[See Table (3a), below for (a,b,c,d) arbitary] $a x^{4}+b y^{4}+c z^{4}=d w^{4}$,

Equation: $a x^{4}+b y^{4}+c z^{4}=w^{4}$

$(4-1-n)$ numerical solution's: $(4<n<50)$

where: $n=(a+b+c)$

Table (3b). $a x^{4}+b y^{4}+c z^{4}=w^{4}$

\begin{tabular}{|c|c|c|c|c|c|c|c|}
\hline$n=(a+b+c)$ & $\mathrm{a}$ & $\mathrm{b}$ & $\mathrm{C}$ & $\mathrm{x}$ & $\mathrm{y}$ & $\mathrm{z}$ & $\mathrm{w}$ \\
\hline [5] & 3 & 1 & 1 & 10 & 17 & 30 & 31 \\
\hline [6] & 4 & 1 & 1 & 2 & 1 & 2 & 3 \\
\hline [7] & 3 & 3 & 1 & 6 & 22 & 5 & 29 \\
\hline$[8]$ & 5 & 2 & 1 & 94 & 40 & 69 & 143 \\
\hline [10] & 5 & 4 & 1 & 20 & 10 & 17 & 31 \\
\hline [11] & 7 & 3 & 1 & 10 & 60 & 3 & 79 \\
\hline [12] & 8 & 3 & 1 & 4 & 6 & 5 & 9 \\
\hline [13] & 1 & 1 & 4 & 35 & 11 & 30 & 39 \\
\hline [14] & 9 & 4 & 1 & 10 & 24 & 17 & 35 \\
\hline [15] & 7 & 7 & 1 & 26 & 38 & 61 & 75 \\
\hline$[16]$ & 8 & 5 & 3 & 1 & 1 & 1 & 2 \\
\hline [17] & 9 & 7 & 1 & 7 & 15 & 11 & 25 \\
\hline$[18]$ & 9 & 7 & 2 & 3 & 1 & 10 & 12 \\
\hline [19] & 9 & 7 & 3 & 1 & 17 & 10 & 28 \\
\hline [20] & 10 & 7 & 3 & 1 & 3 & 2 & 5 \\
\hline [21] & 9 & 7 & 5 & 1 & 1 & 4 & 6 \\
\hline [22] & 13 & 6 & 3 & 3 & 10 & 11 & 18 \\
\hline [23] & 14 & 7 & 2 & 3 & 10 & 19 & 24 \\
\hline [24] & 13 & 8 & 3 & 17 & 22 & 15 & 42 \\
\hline [25] & 11 & 8 & 6 & 1 & 2 & 3 & 5 \\
\hline [26] & 10 & 9 & 7 & 18 & 29 & 59 & 98 \\
\hline [27] & 11 & 9 & 7 & 12 & 25 & 5 & 44 \\
\hline$[28]$ & 11 & 10 & 7 & 2 & 7 & 5 & 13 \\
\hline [29] & 13 & 12 & 4 & 5 & 8 & 9 & 17 \\
\hline [30] & 13 & 11 & 6 & 20 & 15 & 19 & 43 \\
\hline [31] & 14 & 11 & 6 & 2 & 1 & 7 & 11 \\
\hline [32] & 15 & 10 & 7 & 12 & 17 & 15 & 35 \\
\hline [33] & 17 & 10 & 6 & 25 & 2 & 12 & 51 \\
\hline [34] & 18 & 9 & 7 & 10 & 7 & 33 & 54 \\
\hline [35] & 18 & 9 & 8 & 2 & 5 & 3 & 9 \\
\hline [36] & 17 & 15 & 4 & 1 & 3 & 2 & 6 \\
\hline [37] & 31 & 5 & 1 & 2 & 4 & 5 & 7 \\
\hline$[38]$ & 26 & 7 & 5 & 3 & 5 & 2 & 9 \\
\hline [39] & 25 & 8 & 6 & 1 & 5 & 4 & 9 \\
\hline$[40]$ & 22 & 17 & 1 & 2 & 1 & 4 & 5 \\
\hline [41] & 18 & 14 & 9 & 1 & 3 & 2 & 6 \\
\hline$[42]$ & 22 & 19 & 1 & 4 & 2 & 5 & 9 \\
\hline [43] & 30 & 11 & 2 & 1 & 4 & 5 & 8 \\
\hline $\begin{array}{l}{[43]} \\
\end{array}$ & 33 & 8 & 2 & 7 & 16 & 20 & 31 \\
\hline$[44]$ & 24 & 19 & 1 & 4 & 10 & 21 & 25 \\
\hline$[45]$ & 19 & 14 & 12 & 5 & 3 & 6 & 13 \\
\hline$[46]$ & 19 & 17 & 10 & 2 & 3 & 6 & 11 \\
\hline [47] & 43 & 3 & 1 & 14 & 8 & 45 & 49 \\
\hline$[48]$ & 23 & 13 & 12 & 3 & 5 & 1 & 10 \\
\hline [49] & 22 & 19 & 8 & 1 & 3 & 5 & 9 \\
\hline
\end{tabular}


Table 4. (See below) $\left(a x^{4}+b y^{4}+c z^{4}+d w^{4}=0\right)$

\begin{tabular}{|c|c|c|c|c|c|c|c|c|}
\hline Serial nos. & a & $\mathrm{b}$ & $\mathrm{c}$ & d & $\mathrm{x}$ & $\mathrm{y}$ & $\mathrm{z}$ & $\mathrm{w}$ \\
\hline 1 & 6 & -3 & -3 & 1 & 23 & 1 & 47 & 60 \\
\hline 3 & 5 & -2 & -2 & 2 & 4 & 2 & 5 & 1 \\
\hline 4 & 2 & 1 & -1 & 2 & 2 & 3 & 5 & 4 \\
\hline 5 & 4 & 2 & -2 & 1 & 2 & 3 & 37 & 44 \\
\hline 6 & 5 & 4 & -4 & 1 & 20 & 17 & 31 & 40 \\
\hline 7 & 7 & -3 & -3 & 6 & 6 & 5 & 7 & 1 \\
\hline 8 & 5 & 2 & -2 & 3 & 18 & 7 & 23 & 10 \\
\hline 9 & 8 & 6 & -6 & 1 & 10 & 5 & 11 & 8 \\
\hline 10 & 7 & 2 & -2 & 3 & 2 & 1 & 3 & 2 \\
\hline 11 & 8 & 5 & -5 & 3 & 10 & 9 & 13 & 10 \\
\hline 12 & 7 & 5 & -3 & 3 & 4 & 2 & 5 & 1 \\
\hline 13 & 7 & 3 & -3 & 6 & 3 & 13 & 30 & 25 \\
\hline 14 & 11 & 2 & -2 & 3 & 5 & 9 & 10 & 1 \\
\hline 15 & 8 & 4 & -4 & 7 & 26 & 7 & 31 & 8 \\
\hline 16 & 10 & 7 & -2 & 1 & 15 & 11 & 47 & 55 \\
\hline 17 & 10 & 8 & -8 & 7 & 4 & 3 & 5 & 4 \\
\hline 18 & 13 & 3 & -2 & 4 & 5 & 1 & 8 & 2 \\
\hline 19 & 12 & 4 & -2 & 5 & 1 & 5 & 6 & 2 \\
\hline 20 & 8 & 7 & -3 & 8 & 1 & 2 & 4 & 3 \\
\hline 21 & 11 & 5 & -2 & 7 & 5 & 9 & 12 & 4 \\
\hline 22 & 12 & 6 & -5 & 9 & 5 & 8 & 9 & 3 \\
\hline 23 & 9 & 8 & -2 & 8 & 2 & 3 & 10 & 7 \\
\hline 24 & 9 & 8 & -3 & 9 & 5 & 4 & 11 & 7 \\
\hline 25 & 12 & 6 & -3 & 10 & 4 & 1 & 6 & 3 \\
\hline 26 & 13 & 7 & -4 & 10 & 1 & 11 & 15 & 10 \\
\hline 27 & 11 & 7 & -1 & 10 & 2 & 5 & 13 & 7 \\
\hline 28 & 15 & 1 & -1 & 13 & 2 & 3 & 19 & 10 \\
\hline 29 & 12 & 9 & -3 & 11 & 5 & 7 & 10 & 3 \\
\hline
\end{tabular}

\section{Conclusions}

Re: Equation, $\left(\mathrm{ax}^{4}+\mathrm{by}^{4}+\mathrm{cz}^{4}+\mathrm{dw}^{4}\right)=0 \quad---(1)$, while in section (A) we noticed, that when we imposed condition's on Equation (1) like (abcd) $=\square \quad \&$ $((a+b)=(c+16 d))$, we got small numerical solutions for the variable's (X,Y,Z,W) [see table (1)]. But in section (B) in table $(2 a \& 2 b)$ when we used different numerical values of coefficients (a,b,c,d) for Equation (1) a clear relationship has not emerged between the coefficient's (a,b,c,d). For example, in some cases we took, $(\mathrm{a}, \mathrm{b}, \mathrm{c}, \mathrm{d})=(23,23,2,25)=(1,36,27,4)=(49,49,8,1)=(3,75,5,8)$. The interested reader may try to find a pattern in the above (a,b,c,d), which could facilitate discovering more similar identities of the type given for case (1) in section (B) shown below for example:

$$
\begin{array}{r}
7(t+4)^{4}+7(2 t)^{4}+\left(3 t^{2}+24 t-16\right)^{2} \\
=8\left(4 t^{2}+4 t+16\right)^{2}
\end{array}
$$

\section{Acknowledgments}

The authors are thank-full to mathematician Dr. Ajai Choudhry for his comments about this paper.

\section{REFERENCES}

[1] A Note upon an Arithmetical Property of Quartic Surfaces Herbert W. Richmond, vol. s1-23, issue 1, Jan 1948

[2] H. W. Richmond, on the Diophantine Equation, $\left(a x^{4}+\right.$ $\left.b y^{4}+c z^{4}+d w^{4}\right)=0$; The product $(a b c d)$ being a square number, J. London Math. Soc.19 (1944), (193-194).

[3] Constructions of diagonal quartic and sextic surfaces with infinitely many rational points. Preprint, Arxiv 1402, 19 Feb. 2014, Authors: Ajai Choudhry, Andrew Bremner, Maciej Ulas 
[4] Seiji Tomita, webpage, http://www.maroon.dti.ne.jp/ fermat, web site on "Computation number theory" Ref. Article \# 391,397 \& 398. Web page includes listing of published math papers by author.

[5] Ajai Choudhry, the Diophantine Equation $A^{4}+B^{4}=$ $C^{4}+D^{4}$; Indian Journal of Pure and Applied Mathematics, 22 (1991), (9-11).

[6] Oliver Couto, webpage, http://www.celebratingmathematics.com, web site on "Number theory mathematics" Ref. (articles by O. Couto), art \# 12. Web page also has reference to published math papers by author.

[7] Ajai Choudhry, on the Diophantine Equation, $A^{4}+h B^{4}=$ $C^{4}+h D^{4}$ Indian Journal of Pure and Applied Mathematics, (26 (1995), (1057-1061)

[8] J.H. Silverman: Rational points on elliptic curves, arithmetic of elliptic curves, 1992

[9] Ajai Choudhry, The Diophantine Equation, $A^{4}+4 B^{4}=$ $C^{4}+4 D^{4}$, Indian Journal of Pure Applied Mathematics, 29 (1998), (1127-1128).

[10] Jarosław Wróblewski \& A. Choudhry, an Ancient Diophantine Equation with applications to Numerical Curios and Geometric Series.

[11] Ajai Choudhry, on a quartic Diophantine Equation, The Mathematics Student, 70 (2001), (181-183).
[12] Table (3) of numerical solutions for the (4-1-n) Equation, provided by Seiji Tomita via email

[13] Noam Elkies, on eqn. $A^{4}+B^{4}+C^{4}=D^{4}$, Math. Comp. 51 (1988), 825-835.

[14] Crenome's \& Stein-Watkins tables for elliptic curves

[15] Computer software: Pari, Maple \& Magma

[16] Elliptic curves tables: http://www. Imfdb.org

[17] Tito Piezas-online book Collection of Algebraic identities, (Part Seven, Fourth powers) http://sites.google.com/ site/tpiezas

[18] R. D. Carmichael, on certain Diophantine Equations having multiple Parameteric solutions, Amer. Math. Monthly 20 (1913), 304-307.

[19] A-S. Elsenhans, Rational points on diagonal quartic surfaces, Math. Comp. 81 (2012), 481-492.

[20] A. Logan, D. McKinnon, R. Van Luijk, Density of rational points on diagonal quartic surfaces, Algebra and Number Theory 4 (2010), (1-20).

[21] A-S. Elsenhans and J. Jahnel, The diophantine Equation $x^{4}+2 y^{4}=z^{4}+4 w^{4} ; \quad$ Math. Comp. $75 \quad$ (2005), (935-940).

[22] P. Swinnerton-Dyer, Arithmetic of diagonal quartic surfaces, Proc. London Math. Soc. 80 (2000), pages (513-544). 